\title{
Is Intimate Partner Violence a Risk Factor for Cardiovascular Disease in Women? A Review of the Preponderance of the Evidence
}

\author{
Rose Eva Constantino', Alona D. Angosta², Andrew Thomas Reyes ${ }^{2}$, Brayden Kameg1, \\ Linden Wu $\mathbf{u}^{1}$, Jordan Cobb ${ }^{1}$, Vivian Hui ${ }^{1}$, Daisy Palompon ${ }^{3}$, Reema Safadi ${ }^{4}$, \\ Mayada Daibes ${ }^{5}$, Elizabeth Schlenk ${ }^{1}$
}

\author{
${ }^{1}$ Department of Health and Community Systems, School of Nursing, University of Pittsburgh, Pittsburgh, USA \\ ${ }^{2}$ School of Nursing, University of Nevada Las Vegas, Las Vegas, USA \\ ${ }^{3}$ School of Nursing, Cebu Normal University, Cebu City, Philippines \\ ${ }^{4}$ School of Nursing, University of Jordan, Amman, Jordan \\ ${ }^{5}$ Philadelphia University, Amman, Jordan \\ Email: rco100@pitt.edu
}

How to cite this paper: Constantino, R.E., Angosta, A.D., Reyes, A.T., Kameg, B., Wu, L., Cobb, J., Hui, V., Palompon, D., Safadi, R., Daibes, M. and Schlenk, E. (2019) Is Intimate Partner Violence a Risk Factor for Cardiovascular Disease in Women? A Review of the Preponderance of the Evidence. Health, 11, 841-854.

https://doi.org/10.4236/health.2019.116067

Received: May 22, 2019

Accepted: June 23, 2019

Published: June 26, 2019

Copyright $\odot 2019$ by author(s) and Scientific Research Publishing Inc. This work is licensed under the Creative Commons Attribution International License (CC BY 4.0).

http://creativecommons.org/licenses/by/4.0/

c) (i) Open Access

\begin{abstract}
This paper examines the relationship between intimate partner violence (IPV) experience in women and cardiovascular disease (CVD), and determines if there is a preponderance of literature evidence. Research is reviewed dealing with the different biopsychosocial factors affecting the relationship between IPV and CVD. As a result of our review, we propose a framework on the biopsychosocial pathway of IPV as a risk factor of CVD of women. Our proposed framework portrays how IPV experiences contribute to long-term biopsychosocial changes that increase the risk of CVD among female victims of IPV. These biopsychosocial changes include chronic inflammation and hypothalamus-pituitary-adrenal axis dysfunction, metabolic or endocrine dysfunction, and mood symptomatology. In our framework, we also included strategies to prevent risks in developing CVD through the three levels of prevention. Because gender disparities exist when examining CVD risk and development, the correlation between IPV and CVD risk in women must be explored. This framework may provide a theoretical foundation for further research on the relationship between IPV and CVD among women.
\end{abstract}

\section{Keywords}

Intimate Partner Violence, Cardiovascular Disease, Women 


\section{Introduction}

Intimate partner violence (IPV) encompasses abuse across a continuum, including verbal and emotional abuse, reproductive coercion, financial manipulation, sexual or physical assault, imprisonment, humiliation, and stalking with women at high risk for exposure [1] [2]. Each of the aforementioned types of abuse can occur in isolation or in combination with others and results in significant physical, mental, economic, and social sequelae for the survivor. Ultimately, IPV is a major source of morbidity and mortality both globally and across the United States; however, it is grossly underreported [3]. In addition to individual survivor consequences, IPV is correlated with vast societal costs, with estimates of 13.6 to 15.6 billion dollars in expenditures by 2021 and rates of more than 1000 preventable deaths in the United States annually [4].

Survivors of IPV lose a total of nearly 8 million days of paid work, the equivalent of more than 32,000 full-time jobs and nearly 5.6 million days of household productivity each year [5]. Further, residual healthcare expenditures can persist for well over a decade after the cessation of violence exposure [6]. To reduce individual and systemic costs, sequelae of IPV must be explored so that preventive and harm reduction strategies can be implemented as appropriate. The purpose of this paper is to examine whether IPV experience in women and cardiovascular disease exist and if there is a preponderance of literature evidence.

Cardiovascular disease (CVD) is the leading cause of death in women, yet often, signs and symptoms of CVD in this population are not recognized and are thus left under- or untreated [7]. In women, CVD accounts for one of three deaths [8]. While declination in mortality associated with CVD has been noted over the last thirty years, this trend has recently been stagnant, with disparities noted, again, among women [9]. Women are also less likely to receive aggressive treatment to mitigate CVD progression or risk, including adequate use of antihypertensive or statin therapy, engagement in cardiac habilitation or rehabilitation, or utilization of invasive procedures [10] [11] [12].

Empirical evidence shows women not only live longer than men with CVD, but also have worse prognoses and spend approximately twice as many years with the decreased quality of life as measured by loss of productivity, functional limitations, and emotional distress [10]. Clearly, gender disparities exist when examining CVD risk and development. Exacerbating this is the fact that IPV exposure may precipitate CVD development through biopsychosocial processes to include chronic inflammation and hypothalamus-pituitary-adrenal (HPA) axis dysfunction, metabolic or endocrine dysfunction, and mood symptomatology. Because of the gender nuances associated with CVD, the correlation between IPV and CVD risk in women must be explored.

\section{Conceptual Framework: IPV as a Chronic Disease and a CVD Risk Factor}

IPV is considered a chronic disease [13] [14]. Chronic diseases are characterized 
by periods of remission and flare-ups, calm and exacerbations, and episodic recurrence that could go on throughout a lifespan and even generations, a mirror image of the vicious cycle of IPV. The focus in treating chronic diseases is usually not on finding a cure but in health, community, and social health promotion (primary prevention), restoration (secondary prevention) and rehabilitation (tertiary prevention) levels of prevention [15]. The chronicity of IPV lies in the unpredictability of the violent event, the anxiety, anger, and depression that lingers and the cyclical periods of calm and exacerbations beyond the control of the survivor over the behavior and actions of the perpetrator. Further, abuse can occur across a lifelong continuum on a generational basis, spanning from early childhood abuse or neglect to violence exposure in adolescence or adulthood. Figure 1 represents our proposed framework on the biopsychosocial pathway of IPV and CVD and the three levels of prevention.

Scott-Storey [16] developed a conceptual model called IPV as a gendered risk factor for CVD. Investigations into CVD risk factors specific to women have focused on sex-based and biological differences, with little focus on gender-based psychosocial confounders. IPV, including sexual assault, occurs in all age groups of women and is a serious gendered issue affecting up to one-half of all women within their lifetime [17]. Despite beginning evidence that abuse may increase CVD risk in women, the biobehavioral, physiological and psychological pathways linking abuse to CVD have received little attention from researchers and clinicians. Scott-Storey [16] proposed a conceptual model that delineates the pathways by which IPV may increase CVD risk among women. Within the model, lifetime abuse is positioned as a chronic stressor affecting CVD risk through direct and indirect pathways. Directly, IPV experiences can cause long-term biophysical changes within the body, which increase the risk of CVD. Indirectly, smoking, drinking, and overeating, known CVD risk behaviors, are common coping strategies in response to IPV. In addition, women with IPV histories frequently report depressive symptoms and anxiety, which can persist for years after

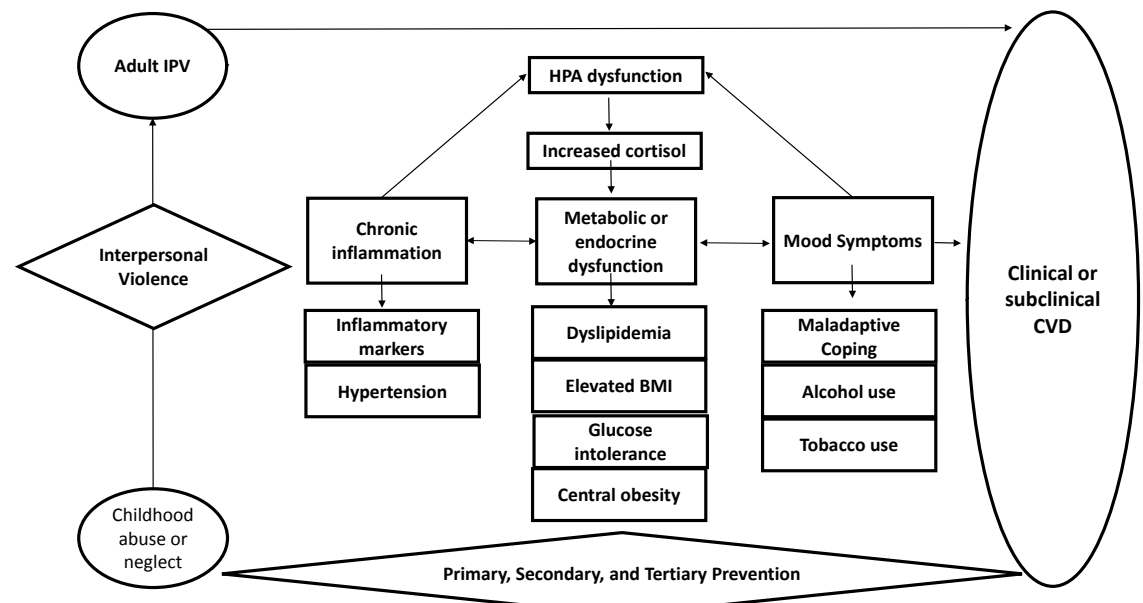

Figure 1. The Constantino, Reyes, Cobb, and Kameg biopsychosocial pathway of IPV and CVD and the three levels of prevention [15]. 
the abusive experience. Depressive symptoms and anxiety are known predictors of CVD and can potentiate CVD risk behaviors. Therefore, depressive symptoms and anxiety are proposed mediators between lifetime IPV and CVD as well as between lifetime IPV and CVD risk behaviors.

Without evidence-based prevention and intervention strategies to reduce CVD risk, morbidity and mortality doubles [18]. Furthermore, the total cost and indirect cost estimates for CVD are higher than for any other major diagnostic categories and is expected to continue as total direct CVD healthcare costs are projected to triple, from $\$ 272.5$ billion to $\$ 818.1$ billion over the next 20 years [19]. Women exposed to IPV in all age groups are an underserved and disadvantaged group due to their focus on fulfilling basic needs, including food, clothing, shelter, and safety for their children and for themselves, rendering them disengaged in their silent, asymptomatic, and preclinical physical health decline. Research shows that physical and sexual abuse in childhood (before 18 years old) is a predictor of early onset of cardiovascular events in adult women. Furthermore, 7 - 14 years of exposure to IPV in late adolescence and young adulthood is associated with increased risk of CVD risk [20].

\section{Chronic Inflammation and Hypothalamus-Pituitary-Adrenal Axis}

It is critical to elucidate the biopsychosocial and physiological pathways that link IPV exposure in adulthood to CVD. It is hypothesized and supported by the current evidence, that such an association is mediated via chronic inflammation, endocrine dysfunction, and indirectly through mood symptomatology and subsequent maladaptive coping strategies. Hall [21] suggests a common denominator that links directly or indirectly all of the numerous lifestyles, emotional, biological, socioeconomic and demographic variables including childhood and intimate partner abuse as risk factors for heart disease is inflammation.

The production of inflammatory molecules may precipitate CVD, while in others, they may be produced in response to the underlying cause. Regardless of whether directly and proximately or indirectly and approximately, inflammation contributes to the gradual loss of cellular energy substrates, the outcomes are the same-impaired diastolic performance. For this reason, Hall [21] argues that referring to a failure of the cardiovascular system as CVD lessens the potentially important contribution of a myriad of other factors. He indicated that impaired cardiac functioning is more than a heart disorder and argues that impaired cardiac functioning can also be a socioeconomic, behavioral, and/or emotional disorder, which subsequently gives rise to metabolic failure.

Violence as a risk factor for CVD influencers is supported by Mason and colleagues [22] when explaining the difference in the prevalence of hypertension when comparing those who experienced emotional abuse versus those who did not, with abuse survivors displaying a $24 \%$ increase in hypertensive status. Further, Newton and colleagues [23] explored inflammatory markers, including C-reactive protein (CRP) and interleukin-6 in women who have been exposed to 
IPV across the lifespan. In women who were exposed to IPV, CRP was likely to be elevated, thus supporting the idea of chronic inflammation in IPV mediates cardiac dysfunction.

There are identified pathways by which IPV is linked to CVD. IPV exposure triggers a cascade of stress-induced biological changes in immune-inflammatory, autonomic, and endocrine responses that elevate CVD risk in later years [24]. In the Lancet study, Tawakol and colleagues [25] investigated the relationship between the amygdala, a region in the brain that is involved in stress, and cardiovascular events in 293 patients (mean age 55 years). They found that amygdalar activity was significantly correlated with arterial inflammation and atherosclerotic calcification and CVD. Activation of this stress network in the brain leads to an increased hematopoietic activity which further leads to arterial inflammation [25].

Chronic stress associated with IPV also stimulates the activation of the sympathetic nervous system and HPA axis which activates the release of CVD biomarkers such as catecholamines, glucocorticoids, and inflammatory cytokines [26] [27] [28]. Finally, in chronic stress syndromes such as posttraumatic stress disorder (PTSD), the HPA axis dysfunction ensues, with increased stress resulting in a mass proliferation of cortisol response, further exacerbating inflammation. Thus, in women who are exposed to repeat/chronic IPV, symptoms of stress and hypervigilance persist, resulting in increased cortisol levels and further fanning inflammatory processes. In summary, a multi-systems approach to primary prevention should be taken to reduce inflammatory processes and subsequent cardiac dysfunction in women in IPV.

\section{Metabolic and Endocrine Dysfunction}

Stress stimulates testosterone response that leads to increased physiological activation and aggressive behaviors [29]. Testosterone, which is also found in females, has also been shown to have a role in the central nervous system involving the perception of threat related to stress or social stimuli [30]. Evidence shows that female victims of IPV have elevated levels of testosterone similar to their male partners who are the aggressors in the relationship [31].

Three hundred forty-two (Black $=114$ and White $=228$ ) women from the Pittsburgh site of the SWAN study were included at baseline assessment of premenopausal or early perimenopausal women in midlife (mean age $=45.7$ ). Women were also evaluated for the presence of the metabolic syndrome over 7 annual follow-up visits. Women were classified as having metabolic syndrome if they met 3 of the following criteria: waist circumference $>88 \mathrm{~cm}$, triglycerides $\geq$ $150 \mathrm{mg} / \mathrm{dl}, \mathrm{HDL}<50 \mathrm{mg} / \mathrm{dl}, \mathrm{SBP} \geq 130$ or DBP $\geq 85 \mathrm{mmHg}$ or on blood pressure medication and fasting glucose $\geq 110 \mathrm{mg} / \mathrm{dl}$ or diabetic [32]. Results showed $34 \%$ of the participants reported a history of physical or sexual childhood abuse [32].

Cox model survival analysis showed that physical abuse was associated with incident metabolic syndrome over the course of 7 years $(\mathrm{HR}=2.12, \mathrm{p}=0.02)$, 
adjusted for ethnicity, age at baseline, and time-dependent menopausal status. Sexual abuse and emotional abuse were unrelated to the metabolic syndrome and that a history of childhood abuse, specifically physical abuse, is related to the development of metabolic syndrome in midlife women. Further, a study conducted by Clark and colleagues [20] demonstrated that women exposed to interpersonal violence, specifically dating violence, were at an increased risk for elevated body mass index (BMI). The risk of elevated BMI was also seen in those who were exposed to childhood trauma. Thus, when women were exposed to both childhood trauma and dating violence, a common co-occurring phenomenon-the risk of elevated BMI could be exacerbated due to the cyclical nature of abuse.

Steptoe \& Kivimaki [33] found early-life stressors, such as childhood abuse and early socioeconomic adversity, are linked to increased cardiovascular morbidity in adulthood. A meta-analysis of prospective studies published until 2011 show a 1.5 -fold (95\% confidence interval $1.2-1.9$ ) increased risk of coronary heart disease among adults experiencing social isolation and a 1.3-fold (1.2 - 1.5) excess risk for workplace stress. Adverse metabolic changes are one of the underlying plausible mechanisms. Stress, anger, and depressed mood can act as acute triggers of major cardiac events; the pooled relative risk of acute coronary syndrome onset being preceded by stress is $2.5(1.8-3.5)$ in case-crossover studies. Stene and colleagues [34] found that women exposed to violence were at-risk for not only low HDL and elevated triglycerides, but also at-risk for engaging in tobacco use behavior; thus demonstrating both physiological, behavioral and psychosocial pathways from violence exposure to cardiac dysfunction. Other maladaptive coping mechanisms that contribute to CVD and which are prevalent in those exposed to violence are further explored below.

\section{Psychosocial Correlates}

To explore psychosocial correlates such as mood symptomatology with coping mechanisms, Batten and colleagues [35] found traumatic experiences in childhood were linked to adult depression and cardiovascular disease. Depression is twice common in women than in men, and depression after cardiovascular events is more common in women than in men. Their data came from the Part 2 sample of the US National Comorbidity Survey, a nationally representative sample containing over 5000 adults. Relationships between childhood maltreatment (sexual abuse, physical abuse, and neglect), adult depression (DSM-III), and CVD were examined using multiple logistic regression models with a specific emphasis on the evaluation of sex differences. Results showed childhood maltreatment was associated with a significant increase in cardiovascular disease for women only and with a significant increase in lifetime depression for both genders. A history of childhood maltreatment removed the natural protection against cardiovascular disease for women and depression for men. Although depression and CVD were correlated, depression did not contribute to the predic- 
tion of CVD in women when controlling for history of childhood maltreatment. They suggest gender is important in evaluating potential psychiatric and physical correlates of childhood maltreatment as maltreatment is a potent risk factor for cardiovascular disease in women and for depression in both women and men. Effective clinical assessment should recognize the role of childhood abuse or neglect in adult health and disease and as far as the authors of this paper are concerned with the role of IPV in adult men and women.

McFarlane and colleagues [36] explored the psychosocial correlates between violence exposure and acute cardiac issues. The aim of the study was to identify a group of economically disadvantaged women while exploring the relationship between a history of physical or sexual abuse and existing symptoms of depression or PTSD. Forty-five women with one of the following conditions were recruited from a large urban hospital: acute myocardial infarction, unstable angina, or angina requiring revascularization. The women completed a demographic questionnaire, the Beck Depression Inventory, and the PTSD Checklist [36]; the latter two were administered at both the three- and six-month follow-ups. At 6 months, women completed the Lifetime Trauma and Victimization History which was modified to be specific to physical or sexual violence. 39 women completed the study at the 6-month follow-up; 25 of the 39 women (64\%) reported a history of abuse. Women who reported abuse were more likely to live alone, more likely to be depressed, and reported more smoking (80\% compared to $29 \%$ ). Regarding mental health issues, women who did not experience abuse reported fewer symptoms of depression and PTSD. Thus, this study highlights that mood symptomatology, including depression or PTSD, acts as a lateral risk factor for the development of maladaptive coping mechanisms which may further exacerbate the risk of cardiac dysfunction.

\section{The Preponderance of the Literature Evidence}

It is documented in the literature that any form of abuse is associated with CVD. Thurston and colleagues [32] investigated the relationship between childhood abuse and CVD risk using subclinical CVD indices among midlife women in the Study of Women's Health across the Nation (SWAN). One of the well-validated measures of subclinical CVD is carotid intima-media thickness (IMT) in which the thickness of the lumen-intima and the media-adventitia interface of the carotid artery and carotid plaque is quantified via ultrasound. They found that childhood sexual abuse was associated with higher intima-media thickness controlling for standard CVD risk factors and other confounders $(p \leq 0.05)$ [32].

Hosang and colleagues [37] examined whether child abuse or neglect was more strongly associated with adult CVD and whether these associations differed by gender using the Childhood Experience of Care and Abuse Questionnaire. Their findings showed that child abuse was significantly associated with adult CVD. Another study by Johnson and colleagues [38] provided an overview of the relationship between toxic stress in children and their high risk for a 
number of health outcomes in adulthood, including CVD and depression. They suggest the neuroendocrine-immune network is altered by early life adversity and how these alterations increase vulnerability to disease. However, they contend even in the most extreme cases of adversity, well-timed changes to children's environments can improve outcomes [39].

Given the cardiotoxic effects of abuse, it is, therefore, reasonable to further investigate. There is limited available evidence in the area of IPV and CVD. O'Neil and Scovelle [24] reported that a positive relationship between IPV and CVD exists. CVD risk factors such as hypertension, carotid atherosclerosis, obesity, hyperlipidemia, smoking, and alcohol consumption were observed in women who were victims of IPV. Furthermore, women who were victims of IPV had coronary heart disease, cardiomyopathy, and suffered from myocardial infarction.

Clark and colleagues [20] investigated IPV exposure in late adolescence and CVD risk later in adulthood through the National Longitudinal Study of Adolescent to Adult Health survey. Data included 99,976 participants, of which 50\% were women. Participants' 30-year risk of CVD was calculated using the Framingham Prediction Model. Their findings revealed that IPV was positively correlated with CVD risk $(p \leq 0.05)$. Stene and colleagues [34] examined the effects of IPV through the Oslo Health Study data. They found that IPV victims had hypertension, abdominal obesity, dyslipidemia, and nicotine dependence compared with women who were not exposed to IPV.

\section{Strategies to Reduce CVD among IPV Survivors}

\subsection{Primary Prevention}

In the three levels of prevention, acting before an event takes place is considered primary prevention. The public health burden of CVD is high in terms of socioeconomic costs. Thurston and colleagues [32] identified key modifiable factors for CVD prevention including health behaviors and health risk factors (e.g., cholesterol, blood pressure). However, a substantial body of research has also identified stress, anxiety, and depression as potentially modifiable CVD risk factors. Mediators appear to be related to chronic inflammation, endocrine dysfunction, and maladaptive coping mechanisms [39]. Thus, primary prevention strategies could individually target each of the aforementioned lateral mediators such as chronic inflammation, metabolic and endocrine dysfunction, or psychiatric correlates. In primary prevention, the strategy of coping with IPV is not proximate or direct, but approximate or indirect because the strategy is implemented way before IPV takes place or it may never take place. For example teaching children and adolescents healthy interpersonal relationships, young mothers' parenting skills or providing a wearable accessory where she can be prompted to walk, exercise or perform a relaxing activity [40].

We suggest Health, Education on safety, and Legal rights and Privileges plus Physical Activity (HELP + PA) or a smart WATCH (Wearable Accessory to Call 
for HELP) as an intervention that leverages biobehavioral and psychosocial factors with diverse universal, selective and indicated population or participants [41] [42] or users. Evidence suggests that email-delivered and Text Messaging-delivered intervention such as HELP best works for women in IPV who may be at risk for depression and low social support [43] [44]. Yoga, meditation, and mindfulness therapy are primary prevention interventions that are effective in reducing stress [45] which may even reverse CVD development. A specific meditation technique, Transcendental Meditation (TM), has been shown to reverse or delay the progression of pathophysiological changes in CVD such as high blood pressure, carotid artery intima-media thickness, myocardial ischemia, and left ventricular hypertrophy [46].

\subsection{Secondary Prevention}

The second level of prevention focuses on early detection and interaction with IPV survivors. The goal of secondary prevention is to reduce the duration, severity, and escalation of IPV. Immediate, proximate and emergent care is provided to the IPV survivor and in some cases, the perpetrator, but never together. Direct and timely contact with IPV survivor is strategic in secondary prevention. The focus is on providing effective treatment of the acute and emergent needs of the IPV survivor and safety. Identification of affected individuals or collateral victims including silent witnesses, such as children, elders is part of secondary prevention [43] [44]. Early screening for CVD is crucial. Clinicians must probe further in their history intake about patients with IPV and CVD risk factors.

\subsection{Tertiary Prevention}

The third level of prevention focuses on the restoration or rehabilitation with the goal of restoring all functions and capacity of the IPV survivor as a productive member of her family, community, and society and reduces consequences of the IPV experience [43] [44]. In IPV, tertiary prevention strategies include accurate evaluation of the IPV survivor's ecological space including family, workplace, referrals and community resources.

The aforementioned prevention and intervention strategies do not only help women who are victims of IPV but could help clinicians identify gaps where new or different internet-, mobile or wearable-delivered interventions are explored. Researchers could engage individual participants in the research process as each participant could tailor-shape their own prevention or intervention program guided by their own motivation and preference to take action to fit each participant's capacity for adhering to the activities required to complete each individualized and tailor-made. Furthermore, with breakthroughs in wearable health accessories such as a smartwatch that tracks activities, heart rate, blood pressure, calories, prompts coping or easing symptoms, there is a great future ahead in interfacing technology and primary prevention. Researchers could engage individual participants in the research process as each participant could tailor-shape 
their own prevention or intervention program guided by their own motivation and preference to take action to fit each participant's capacity for adhering to the activities required to complete each individualized and tailor-made program [44] [45].

\section{Implications and Conclusions}

As various aspects of identities intersect, women who are exposed to IPV may face doubling disparities, including low socioeconomic or minority status, which may further predispose individuals to engage in maladaptive coping mechanisms as a means of stress reduction. Further, those without the economic or social resources to fully engage in health promotion activities may need additional support to achieve wellness when compared to their privileged or non-violence exposed peers [46]. Nonetheless, it is critical for both researchers and clinicians to be fully engaged in the identification of biopsychosocial mediating factors towards CVD development, and strategies to mitigate such risk factors, respectively.

Research has clearly highlighted associations between IPV via the cyclical nature of abuse, learned helplessness, and maladaptive coping. Furthermore, evidence highlights IPV as an independent risk factor for CVD via chronic inflammation, dysfunction of HPA, metabolic and endocrine pathways, and mood symptomatology. Thus, the trajectory of evidence is moving towards the elucidation of a definitive association between IPV and CVD, however, more research is warranted and this acts as a significant implication. Nurses, advanced practice nurses, and other clinicians must support women who have been exposed to IPV and other types of violence, not only to optimal psychosocial wellness but also through the mitigation of cardiovascular risk factors.

We have highlighted the preponderance of the literature evidence of the link between IPV and CVD and literature has shown how life experiences can exacerbate or contribute to the development of CVD risks such as inflammation and chronic health conditions, metabolic and endocrine dysfunction, and psychosocial correlates. Diverse demographic groups with unique backgrounds referred to as universal, selective and indicated [40] [47] should be targeted and identified for future research pertaining to violence exposure risk and CVD development. These groups could include lesbian, bisexual, or transgender women, immigrants or refugees, homeless women, and mother-child dyads who have been exposed to violence. The previously described theoretical framework can be modified to meet the needs of unique populations, ultimately promoting optimum health and psychosocial outcomes amongst a variety of groups, and leveling disparities among women with various components of identity that may predispose them to health and social inequities. We anticipate that by 2024, the literature on the proximate relationship between IPV and CVD risks could be proven not only by a preponderance of the evidence but beyond a reasonable doubt. 


\section{Conflicts of Interest}

The authors declare no conflicts of interest regarding the publication of this paper.

\section{References}

[1] Laughon, K. and Campbell, J.C. (2012) Understanding and Addressing Violence against Women: Intimate Partner Violence. World Health Organization, Geneva.

[2] Tjaden, P. and Thoennes, N. (2000) Extent, Nature, and Consequences of Intimate Partner Violence: Findings from the National Violence against Women Survey. National Institute of Justice and the Center for Disease Control and Prevention, Washington DC.

[3] García-Moreno, C., Pallitto, C., Devries, K., Stöckl, H., Watts, C. and Abrahams, N. (2013) Global and Regional Estimates of Violence against Women: Prevalence and Health Effects of Intimate Partner Violence and Non-Partner Sexual Violence. World Health Organization, Geneva. https://doi.org/10.1161/CIRCRESAHA.116.307547

[4] Dolezal, T., McCollum, D. and Callahan, M. (2009) Hidden Costs in Health Care: The Economic Impact of Violence and Abuse. The Academy on Violence and Abuse, Eden Prairie.

[5] Centers for Disease Control and Prevention (2017) Intimate Partner Violence: Consequences. Center for Disease Control and Prevention, Atlanta.

[6] Rivara, F.P., Anderson, M.L., Fishman, P., Bonomi, A.E., Reid, R.J., Carrell, D. and Thompson, R.S. (2007) Healthcare Utilization and Costs for Women with a History of Intimate Partner Violence. American Journal of Preventive Medicine, 32, 89-96. https://doi.org/10.1016/j.amepre.2006.10.001

[7] Mozaffarian, D., Benjamin, E.J., Go, A.S., et al. (2015) Heart Disease and Stroke Statistics-2015 Update: A Report from the American Heart Association. Circulation, 131, e29-e322. https://doi.org/10.1161/CIR.0000000000000152

[8] American Heart Association (2018) Heart Disease and Stroke Statistics-2018 Update. https://www.ahajournals.org/doi/pdf/10.1161/CIR.0000000000000558

[9] Mosca, L., Mochari-Greenberger, H., Dolor, R.J., Newby, L.K. and Robb, K.J. (2010) Twelve-Year Follow-Up of American Women's Awareness of Cardiovascular Disease Risk and Barriers to Heart Health. Circulation: Cardiovascular Quality and Outcomes, 109, 120-127. https://doi.org/10.1161/CIRCOUTCOMES.109.915538

[10] Garcia, M., Mulvagh, S.L., Merz, C.N.B., Buring, J.E. and Manson, J.E. (2016) Cardiovascular Disease in Women: Clinical Perspectives. Circulation Research, 118, 1273-1293. https://doi.org/10.1161/CIRCRESAHA.116.307547

[11] Gu, Q., Burt, V.L., Paulose-Ram, R. and Dillon, C.F. (2008) Gender Differences in Hypertension Treatment, Drug Utilization Patterns, and Blood Pressure Control among US Adults with Hypertension: Data from the National Health and Nutrition Examination Survey 1999-2004. American Journal of Hypertension, 21, 789-798. https://doi.org/10.1038/ajh.2008.185

[12] Suaya, J.A., Shepard, D.S., Normand, S.L.T., Ades, P.A., Prottas, J. and Stason, W.B. (2007) Use of Cardiac Rehabilitation by Medicare Beneficiaries after Myocardial Infarction or Coronary Bypass Surgery. Circulation, 116, 1653-1662. https://doi.org/10.1161/CIRCULATIONAHA.107.701466

[13] Lachs, M.S. and Pillemer, K. (1995) Abuse and Neglect of Elderly Persons. New England Journal of Medicine, 332, 437-443. https://doi.org/10.1056/NEJM199502163320706 
[14] Breiding, M.J., Black, M.C. and Ryan, G.W. (2008) Chronic Disease and Health Risk Behaviors Associated with Intimate Partner Violence-18 US States/Territories, 2005. Annals of Epidemiology, 18, 538-544. https://doi.org/10.1016/j.annepidem.2008.02.005

[15] Constantino, R.E., Reyes, A.T., Cobb, J. and Kameg, B. (2019) The Constantino, Reyes, Cobb, and Kameg Biopsychosocial Pathway of IPV and CVD and the Three Levels of Prevention. An Unpublished Manuscript, University of Pittsburgh School of Nursing, Department of Health and Community Systems, Pittsburgh.

[16] Scott-Storey, K.A. (2013) Abuse as a Gendered Risk Factor for Cardiovascular Disease: A Conceptual Model. Journal of Cardiovascular Nursing, 28, E1-E8. https://doi.org/10.1097/JCN.0b013e318279e372

[17] Breiding, M.J. (2014) Prevalence and Characteristics of Sexual Violence, Stalking, and Intimate Partner Violence Victimization-National Intimate Partner and Sexual Violence Survey, United States, 2011. Morbidity and Mortality Weekly Report, 63, 1-18. https://www.cdc.gov/mmwr/preview/mmwrhtml/ss6308a1.htm

[18] Rogers, L.K. and Velten, M. (2011) Maternal Inflammation, Growth Retardation, and Preterm Birth: Insights into Adult Cardiovascular Disease. Life Sciences, 89, 417-421. https://doi.org/10.1016/j.lfs.2011.07.017

[19] Bosak, K. and Martin, L. (2014) Neuroimaging of Goal-Directed Behavior in Midlife Women. Nursing Research, 63, 388-396. https://doi.org/10.1097/NNR.0000000000000060

[20] Clark, C.J., Spencer, R.A., Everson-Rose, S.A., et al. (2014) Dating Violence, Childhood Maltreatment, and BMI from Adolescence to Young Adulthood. Pediatrics, 134, 678-685. https://doi.org/10.1542/peds.2014-1179

[21] Hall, N.R. (2013) Diagnosing Cardiovascular Disease from the Perspective of the Brain and Inflammation. Alternative Therapies in Health and Medicine, 19, 8-9. https://doi.org/10.1089/act.2013.19103

[22] Mason, S.M., Wright, R.J., Hibert, E.N., Spiegelman, D., Forman, J.P. and Rich-Edwards, J.W. (2012) Intimate Partner Violence and Incidence of Hypertension in Women. Annals of Epidemiology, 22, 562-567. https://doi.org/10.1016/j.annepidem.2012.05.003

[23] Newton, T.L., Fernandez-Botran, R., Miller, J.J., Lorenz, D.J., Burns, V.E. and Fleming, K.N. (2011) Markers of Inflammation in Midlife Women with Intimate Partner Violence Histories. Journal of Women's Health, 20, 1871-1880. https://doi.org/10.1037/a0029157

[24] O’Neil, A. and Scovelle, A.J. (2018) Intimate Partner Violence Perpetration and Cardiovascular Risk: A Systematic Review. Preventive Medicine Reports, 10, 15-19. https://doi.org/10.1016/j.pmedr.2018.01.006

[25] Tawakol A., Ishai, A., Takx, A.P., et al. (2017) Relation between Resting Amygdalar Activity and Cardiovascular Events: A Longitudinal and Cohort Study. The Lancet, 389, 834-845. https://doi.org/10.1016/S0140-6736(16)31714-7

[26] De Bosscher, K., Van Craenenbroeck, K., Meijer, O.C. and Haegeman, G. (2008) Selective Transrepression versus Transactivation Mechanisms by Glucocorticoid Receptor Modulators in Stress and Immune Systems. European Journal of Pharmacology, 583, 290-302. https://doi.org/10.1016/j.ejphar.2007.11.076

[27] Nikkheslat, N., Zunszain, P.A., Horowitz, M.A., et al. (2015) Insufficient Glucocorticoid Signaling and Elevated Inflammation in Coronary Heart Disease Patients with Comorbid Depression. Brain, Behavior, and Immunity, 48, 8-18.

https://doi.org/10.1016/j.bbi.2015.02.002 
[28] Miller, A.H., Pariante, C.M. and Pearce, B.D. (1999) Effects of Cytokines on Glucocorticoid Receptor Expression and Function. Glucocorticoid Resistance and Relevance to Depression. Advance in Experimental Medicine and Biology, 461, 107-116. https://doi.org/10.1007/978-0-585-37970-8_7

[29] Porges, E.C., Smith, K.E. and Decety, J. (2015) Individual Differences in Vagal Regulation Are Related to Testosterone Responses to Observed Violence. Frontiers in Psychology, 6, 19. https://doi.org/10.3389/fpsyg.2015.00019

[30] Denson, T.F., O'Dean, S.M., Blake, K. and Beames, J.R. (2018) Aggression in Women: Behavior, Brains, and Hormones. Frontiers in Behavioral Neuroscience, 12, 81. https://www.frontiersin.org/articles/10.3389/fnbeh.2018.00081/full https://doi.org/10.3389/fnbeh.2018.00081

[31] Cohan, C.L., Booth, A. and Granger, D.A. (2003) Gender Moderates the Relationship between Testosterone and Marital Interaction. Journal of Family Psychology, 17, 29-40. https://doi.org/10.1037/0893-3200.17.1.29

[32] Thurston, R.C., Chang, Y., Derby, C.A., Bromberger, J.T., Harlow, S.D., Janssen, I. and Matthews, K.A. (2014) Abuse and Subclinical Cardiovascular Disease among Midlife Women: The Study of Women's Health across the Nation. Stroke, 45, 2246-2251. https://doi.org/10.1161/STROKEAHA.114.005928

[33] Steptoe, A. and Kivimäki, M. (2013) Stress and Cardiovascular Disease: An Update on Current Knowledge. Annual Review of Public Health, 34, 337-354. https://doi.org/10.1146/annurev-publhealth-031912-114452

[34] Stene, L.E., Dyb, G., Tverdal, A., Jacobsen, G.W. and Schei, B. (2012) Intimate Partner Violence and Prescription of Potentially Addictive Drugs: Prospective Cohort Study of Women in the Oslo Health Study. British Medical Journal Open, 2, e000614. https://doi.org/10.1136/bmjopen-2011-000614

[35] Batten, S.V., Aslan, M., Maciejewski, P.K. and Mazure, C.M. (2004) Childhood Maltreatment as a Risk Factor for Adult Cardiovascular Disease and Depression. Journal of Clinical Psychiatry, 65, 249-254. https://doi.org/10.4088/JCP.v65n0217

[36] McFarlane, J., Symes, L., Frazier, L., McGlory, G., Henderson-Everhardus, M.C., Watson, K. and Liu, Y. (2010) Connecting the Dots of Heart Disease, Poor Mental Health, and Abuse to Understand Gender Disparities and Promote Women's Health: A Prospective Cohort Analysis. Health Care for Women International, 31, 313-326. https://doi.org/10.1080/07399330902893853

[37] Hosang, G.M., Johnson, S.L., Kiecolt-Glaser, J., et al. (2013) Gender Specific Association of Child Abuse and Adult Cardiovascular Disease in a Sample of Patients with Basal Cell Carcinoma. Child Abuse \& Neglect, 37, 374-379. https://doi.org/10.1016/j.chiabu.2012.09.018

[38] Johnson, S.B., Riley, A.W., Granger, D.A. and Riis, J. (2013) The Science of Early Life Toxic Stress for Pediatric Practice and Advocacy. Pediatrics, 131, 319-327. https://doi.org/10.1542/peds.2012-0469

[39] Midei, A.J., Matthews, K.A., Chang, Y.F. and Bromberger, J.T. (2013) Childhood Physical Abuse Is Associated with Incident of Metabolic Syndrome in Mid-Life Women. Health Psychology, 32, 121-127. https://doi.org/10.1037/a0027891

[40] Constantino, R. and Privitera, M.R. (2011) Prevention Terminology: Primary, Secondary, Tertiary and Evolution of Terms. In: Privitera, M.R., Ed., Workplace Violence in Mental and General Health Care Settings, Jones and Bartlett, Boston, 15-22.

[41] Gordon, R. (1983) An Operational Classification of Disease Prevention. Public Health Reports, 98, 107-109. 
[42] Institute of Medicine (2004) Reducing Risks for Mental Disorders: Frontiers of Preventive Intervention Research. National Academy Press, Washington DC.

[43] Constantino, R., Wu, L., de la Cruz, D., Burroughs, J., Hwang, J.H., Henderson, A. and Braxter, B. (2014) Exploring the Feasibility of Text Messaging Intervention in Intimate Partner Violence. Open Journal of Nursing, 4, 528-537. https://doi.org/10.4236/ojn.2014.47056

[44] Constantino, R.E., Braxter, B., Ren, D., Burroughs, J.D., Doswell, W., Wu, L. and Greene, W.B. (2015) Comparing Online with Face-to-Face HELPP Intervention in Women Experiencing Intimate Partner Violence. Issues in Mental Health Nursing, 36, 430-438. https://doi.org/10.3109/01612840.2014.991049

[45] Ramadoss, R. and Bose, B. (2010) Transformative Life Skills: Pilot Study of a Yoga Model for Stress and Improving Self-Control in Vulnerable Youth. International Journal of Yoga, 20, 73-78.

[46] Walton, K.G., Schneider, R.H., Nidich, S.I., Salemo, J.W., Nordstrom, C.K. and Merz, C.N.B. (2002) Psychosocial Stress and Cardiovascular Disease Part 2: Effectiveness of the Transcendental Meditation Program in Treatment and Prevention. Behavioral Medicine, 28, 106-123. https://doi.org/10.1080/08964280209596049

[47] Reyes, A.T. and Constantino, R.E. (2016) Asian American Women's Resilience: An Integrative Review. Asian/ Pacific Island Nursing Journal, 1, 105-115.

https://doi.org/10.9741/23736658.1048

\section{Abbreviation Note List}

BMI: Body Mass Index

CRP: Creatinine-Reactive Protein

CVD: Cardiovascular Disease

DSM-III: Diagnostic and Statistical Manual of Mental Disorders-III

IMT: Intima-Media Thickness

IPV: Intimate Partner Violence

HELP + PA: Health Education, Legal rights, and Privileges + Physical Activity

HDL: High-Density Lipoprotein

HPA: Hypothalamus-Pituitary-Adrenal

PTSD: Posttraumatic Stress Disorder

SWAN: Study of Women's Health across the Nation

WATCH: Wearable Accessory to Call for HELP 